\title{
A New Class of Adiabatic Cyclic States and Geometric Phases for Non-Hermitian Hamiltonians
}

\author{
Ali Mostafazadeh* \\ Department of Mathematics, Koç University, \\ Istinye 80860, Istanbul, TURKEY
}

\begin{abstract}
For a $T$-periodic non-Hermitian Hamiltonian $H(t)$, we construct a class of adiabatic cyclic states of period $T$ which are not eigenstates of the initial Hamiltonian $H(0)$. We show that the corresponding adiabatic geometric phase angles are real and discuss their relationship with the conventional complex adiabatic geometric phase angles. We present a detailed calculation of the new adiabatic cyclic states and their geometric phases for a non-Hermitian analog of the spin $1 / 2$ particle in a precessing magnetic field.
\end{abstract}

Since the publication of Berry's paper [1] on the adiabatic geometrical phase, the subject has undergone a rapid development. Berry's adiabatic geometric phase for periodic Hermitian Hamiltonians with a discrete nondegenerate spectrum has been generalized to arbitrary changes of a quantum state. In particular, the conditions on the adiabaticity [2] and cyclicity [3], 4, 5] of the evolution, Hermiticity of the Hamiltonian [6], and degeneracy [7] and discreteness [8] of its spectrum have been lifted. Moreover, the classical [9] and relativistic [10] analogues of the geometric phase have been considered.

The purpose of this note is to offer an alternative generalization of Berry's treatment of the adiabatic geometric phase for a non-Hermitian parametric Hamiltonian $H[R]$ with a nondegenerate discrete spectrum. The parameters $R=\left(R^{1}, \cdots, R^{n}\right)$ are assumed to be real

\footnotetext{
${ }^{*}$ E-mail address: amostafazadeh@ku.edu.tr
} 
coordinates of a smooth parameter manifold, with the eigenvalues $E_{n}[R]$ and eigenvectors $\left|\psi_{n}, R\right\rangle$ of $H[R]$ depending smoothly on $R$. The approach pursued in the present paper differs from those of Refs. [6, 11, 12, 13] in the choice of the adiabatically evolving state vectors. The corresponding geometric phase angle, which is shown to be real, has the same expression as the geometric phase angle for a Hermitian Hamiltonian. It differs from the conventional complex geometric phase angle by terms which are small for an adiabatically evolving system. In particular, this implies that in a generic adiabatic evolution the imaginary part of the complex adiabatic geometric phase angle is small.

The generalization of the results of Berry [1] to non-Hermitian Hamiltonians was originally considered by Garrison and Wright [6] and further developed by Dattoli et al [11], Miniatura et al [12], and Mondragón and Hernandez [13]. The main ingredient used by all of these authors in their derivation of a non-Hermitian analog of Berry's phase is the biorthonormal eigenbasis of the Hamiltonian. More specifically, one writes the evolving state vector $|\psi(t)\rangle$ in an eigenbasis $\left|\psi_{n}, t\right\rangle:=\left|\psi_{n}, R(t)\right\rangle$ of the Hamiltonian $H(t):=H[R(t)]$,

$$
|\psi(t)\rangle=\sum_{n} C_{n}(t)\left|\psi_{n}, t\right\rangle,
$$

and enforces the Schrödinger equation

$$
i|\dot{\psi}(t)\rangle=H(t)|\psi(t)\rangle
$$

where $R(t)$ denotes the curve traced in the parameter space, $C_{n}(t)$ are complex coefficients, a dot denotes a time-derivative, and $\hbar$ is set to 1 . The resulting equation together with the eigenvalue equation for the Hamiltonian,

$$
H[R]\left|\psi_{n}, R\right\rangle=E_{n}[R]\left|\psi_{n}, R\right\rangle
$$

where $R=R(t)$, yield

$$
\sum_{n}\left[i C_{n}(t)\left|\dot{\psi}_{n}, t\right\rangle+\left[i \dot{C}_{n}(t)-E_{n}(t) C_{n}(t)\right]\left|\psi_{n}, t\right\rangle\right]=0 .
$$

Next one takes the inner product of both sides of Eq. (4) with the eigenvectors $\left|\phi_{n}, t\right\rangle=$ $\left|\phi_{n}, R(t)\right\rangle$ of $H^{\dagger}(t)=H^{\dagger}[R(t)]$ which are defined by

$$
\begin{aligned}
& H^{\dagger}[R]\left|\phi_{n}, R\right\rangle=E_{n}^{*}[R]\left|\phi_{n}, R\right\rangle, \\
& \left\langle\phi_{m}, R \mid \psi_{n}, R\right\rangle=\delta_{m n} .
\end{aligned}
$$


One then finds

$$
i C_{m}(t)\left\langle\phi_{m}, t \mid \dot{\psi}_{m}, t\right\rangle+i \dot{C}_{m}(t)-E_{m}(t) C_{m}(t)+\sum_{n \neq m} i C_{n}(t)\left\langle\phi_{m}, t \mid \dot{\psi}_{n}, t\right\rangle=0 .
$$

For an adiabatically changing Hamiltonian,

$$
\left\langle\phi_{m}, t \mid \dot{\psi}_{n}, t\right\rangle=\frac{\left\langle\phi_{m}, t|\dot{H}(t)| \psi_{n}, t\right\rangle}{E_{n}(t)-E_{m}(t)}, \quad \text { for } \quad m \neq n
$$

are small and the sum in (7) may be neglected [6, 14]. In this case, Eqs. (7) can be easily integrated to yield

$$
\begin{aligned}
C_{m}(t) & \approx C_{m}(0) e^{i\left[\delta_{m}(t)+\gamma_{m}(t)\right]} \\
\delta_{m}(t) & :=-\int_{0}^{t} E_{m}\left(t^{\prime}\right) d t^{\prime} \\
\gamma_{m}(t) & :=\int_{0}^{t} i\left\langle\phi_{m}, t^{\prime} \mid \dot{\psi}_{m}, t^{\prime}\right\rangle d t^{\prime}
\end{aligned}
$$

The validity of the adiabatic approximation, i.e., Eq. (9), is measured by the value of the adiabaticity parameter which is defined by

$$
\eta:=\frac{1}{\omega_{0}} \operatorname{Sup}_{n, m \neq n, t}\left(\left|\left\langle\phi_{m}, t \mid \dot{\psi}_{n}, t\right\rangle\right|\right)
$$

Here 'Sup' stands for 'Supremum' and $\omega_{0}$ is the frequency (energy) scale of the system, [15]. Adiabatic approximation is valid if and only if $\eta \ll 1$. It is exact if and only if $\eta=0$.

For a periodic Hamiltonian with period $T$, where $R(T)=R(0)$, the eigenvectors of the initial Hamiltonian undergo approximate cyclic evolutions, i.e., for $|\psi(0)\rangle=\left|\psi_{n}, 0\right\rangle$,

$$
|\psi(T)\rangle \approx e^{i\left[\delta_{m}(T)+\gamma_{m}(T)\right]}\left|\psi_{n}, 0\right\rangle
$$

In this case $\delta_{m}(T)$ and $\gamma_{m}(T)$ are called the adiabatic dynamical and geometrical phase angles, respectively. The geometric phase angle $\gamma_{m}(T)$ which can also be expressed in the form

$$
\gamma_{m}(T)=\oint i\left\langle\phi_{m}, R|d| \psi_{m}, R\right\rangle
$$

is the complex analogue of Berry's adiabatic geometrical phase angle [1]. In Eq. (14), $d$ stands for the exterior derivative with respect to the parameters $R^{i}$. 
The approximate equations (91) and (13) tend to exact equations only in the extreme adiabatic limit, $\eta=0$, where the eigenvectors of the Hamiltonian become stationary and the adiabatic approximation is exact [16]. In this case, however, the geometric phase angle (14) vanishes identically. For a system with nonstationary energy eigenstates, $\eta \neq 0$, and the adiabatic approximation is never exact [16]. In this case the eigenstates of the initial Hamiltonian are only approximately cyclic. Strictly speaking, the state represented by $|\psi(T)\rangle$ lies in a neighborhood of the state represented by $|\psi(0)\rangle$ whose radius is of order $\eta$.

The main motivation for the present analysis is the fact that although (due to the adiabaticity of the evolution) the components of $\left|\dot{\psi}_{m}, t\right\rangle$ along the 'normal' directions to $\left|\psi_{n}, t\right\rangle$ are negligible, $\left|\dot{\psi}_{m}, t\right\rangle$ does have non-negligible components along other eigenvectors. This is mainly because for a non-Hermitian Hamiltonian $\left|\psi_{m}, t\right\rangle$ do not form an orthogonal basis. This observation suggests an alternative expansion for the evolving state $|\psi(t)\rangle$, namely

$$
|\psi(t)\rangle=\sum_{n \neq m} C_{n}(t)\left|\psi_{n}, t\right\rangle+\tilde{C}(t)\left|\phi_{m}, t\right\rangle=\tilde{C}(t)\left[\sum_{n \neq m} \tilde{C}_{n}(t)\left|\psi_{n}, t\right\rangle+\left|\phi_{m}, t\right\rangle\right] .
$$

Here $m$ is a fixed label, $C_{n}(t)$ and $\tilde{C}(t)$ are complex coefficients, $\tilde{C}_{n}:=C_{n} / \tilde{C}$, and $\tilde{C}(t)$ is assumed not to vanish.

Although the expansions (四) and (15) are mathematically equivalent, the latter allows for the construction of a new class of adiabatic cyclic states of period $T$ which are not eigenstates of the initial Hamiltonian. It should be emphasized that these states also have approximate cyclic evolutions. More specifically after each complete period $T$ of the Hamiltonian, the corresponding final state lies in a neighborhood of the initial state with the radius of the neighborhood being of order $\eta$.

Substituting (15) in the Schrödinger equation (2), taking the inner product of both sides of the resulting equation first with $\left|\phi_{m}, t\right\rangle$ and then with $\left|\psi_{k}, t\right\rangle$ for $k \neq m$, and enforcing the adiabaticity condition: $\eta \ll 1$ which means

$$
\left\langle\phi_{m}, t \mid \dot{\psi}_{n}, t\right\rangle \approx 0 \approx\left\langle\psi_{k}, t \mid \dot{\phi}_{m}, t\right\rangle, \quad \text { for } \quad n \neq m \neq k,
$$

\footnotetext{
${ }^{1}$ The states belong to the projective Hilbert space which is endowed with the Fubini-Study metric or its infinite-dimensional analog. The latter is used to define the notion of a neighborhood of a state.
} 
one obtains

$$
\begin{gathered}
i\left\langle\phi_{m}, t \mid \phi_{m}, t\right\rangle \dot{\tilde{C}}(t)+\left[-\left\langle\phi_{m}, t \mid \phi_{m}, t\right\rangle E_{m}(t)+i\left\langle\phi_{m}, t \mid \dot{\phi}_{m}, t\right\rangle\right] \tilde{C}(t) \approx 0 \\
\sum_{n \neq m}\left\{\left\langle\psi_{k}, t \mid \psi_{n}, t\right\rangle\left[\dot{\tilde{C}}_{n}(t)+\left(i E_{n}(t)+\frac{\dot{\tilde{C}}(t)}{\tilde{C}(t)}\right) \tilde{C}_{n}\right]+\left\langle\psi_{k}, t \mid \dot{\psi}_{n}, t\right\rangle \tilde{C}_{n}(t)\right\} \\
\approx-i\left\langle\psi_{k}, t|H(t)| \phi_{m}, t\right\rangle, \text { for } k \neq n
\end{gathered}
$$

Eq. (17) can be immediately integrated to yield

$$
\begin{aligned}
\tilde{C}(t) & \approx \tilde{C}(0) e^{i\left[\tilde{\delta}_{m}(t)+\tilde{\gamma}_{m}(t)\right]} \\
\tilde{\delta}_{m}(t) & :=-\int_{0}^{t} E_{m}\left(t^{\prime}\right) d t^{\prime} \\
\tilde{\gamma}_{m}(t) & :=\int_{0}^{t} \frac{i\left\langle\phi_{m}, t^{\prime} \mid \dot{\phi}_{m}, t^{\prime}\right\rangle}{\left\langle\phi_{m}, t^{\prime} \mid \phi_{m}, t^{\prime}\right\rangle} d t^{\prime}
\end{aligned}
$$

Using Eq. (17), one can write Eq. (18) in the form:

$$
\begin{gathered}
\sum_{n \neq m}\left\{\left\langle\psi_{k}, t \mid \psi_{n}, t\right\rangle\left[\dot{\tilde{C}}_{n}(t)+i\left(E_{n}(t)-E_{m}(t)+\frac{i\left\langle\phi_{m}, t \mid \dot{\phi}_{m}, t\right\rangle}{\left\langle\phi_{m}, t \mid \phi_{m}, t\right\rangle}\right) \tilde{C}_{n}(t)\right]+\left\langle\psi_{k}, t \mid \dot{\psi}_{n}, t\right\rangle \tilde{C}_{n}(t)\right\} \\
\approx-i\left\langle\psi_{k}, t|H(t)| \phi_{m}, t\right\rangle \quad \text { for } \quad k \neq n .
\end{gathered}
$$

This is a system of first order linear ordinary differential equations with $T$-periodic coefficients. If the initial conditions are such that its solution is $T$-periodic, i.e., $\tilde{C}_{n}(T) \approx \tilde{C}_{n}(0)$, then the evolving state undergoes an approximate cyclic evolution,

$$
|\psi(T)\rangle \approx e^{i\left[\tilde{\delta}_{m}(T)+\tilde{\gamma}_{m}(T)\right]}|\psi(0)\rangle
$$

As seen from Eq. (23), the dynamical part of the total (complex) phase angle, namely $\tilde{\delta}_{m}(T)$ has the same form as (10), but the geometric part which can be written in the form

$$
\tilde{\gamma}_{m}(T)=\oint \frac{i\left\langle\phi_{m}, R \mid d \phi_{m}, R\right\rangle}{\left\langle\phi_{m}, R \mid \phi_{m}, R\right\rangle},
$$

differs from (14). An interesting property of this geometric phase angle is that it has the same form as the geometric phase angle for a Hermitian Hamiltonian, [1]. It is also very easy to show that $\tilde{\gamma}_{m}(T)$ is real.

We wish to emphasize that the (cyclic) geometric phase obtained here is a physical quantity, if Eq. (22) has a periodic solution. Although this is a linear first order system with 
$T$-periodic coefficients, its solutions are not generally $T$-periodic [17]. In the following, we shall first elaborate on the relationship between the conventional complex geometric phase angle (14) and our real geometric phase angle (21). We shall then explore the condition of the existence of periodic solutions of (22) for the simplest nontrivial case, i.e., the two-level system.

We first introduce

$$
\begin{aligned}
& \mathcal{A}_{m n}(t):=i\left\langle\phi_{m}, t \mid \dot{\psi}_{n}, t\right\rangle, \quad \tilde{\mathcal{A}}_{m n}(t):=\frac{i\left\langle\phi_{m}, t \mid \dot{\phi}_{n}, t\right\rangle}{\left\langle\phi_{n}, t \mid \phi_{n}, t\right\rangle}, \\
& \mathcal{A}_{m}(t):=\mathcal{A}_{m m}(t), \quad \tilde{\mathcal{A}}_{m}(t):=\tilde{\mathcal{A}}_{m m}(t)
\end{aligned}
$$

so that

$$
\gamma_{m}(t)=\int_{0}^{t} \mathcal{A}_{m}\left(t^{\prime}\right) d t^{\prime}, \quad \text { and } \quad \tilde{\gamma}_{m}(t)=\int_{0}^{t} \tilde{\mathcal{A}}_{m}\left(t^{\prime}\right) d t^{\prime} .
$$

Now using the completeness of the biorthobormal basis vector, i.e., $\sum_{n}\left|\psi_{n}, t\right\rangle\left\langle\phi_{n}, t\right|=1$, we can write $\left|\phi_{m}, t\right\rangle=\sum_{n}\left\langle\phi_{n}, t \mid \phi_{m}, t\right\rangle\left|\psi_{n}, t\right\rangle$. Substituting this relation in the definition of $\tilde{\mathcal{A}}_{m}(t)$ and performing the necessary algebra, we obtain

$$
\tilde{\mathcal{A}}_{m}(t)=\mathcal{A}_{m}(t)+i \frac{d}{d t} \ln \left\langle\phi_{m}, t \mid \phi_{m}, t\right\rangle+\sum_{n \neq m}\left(\frac{\left\langle\phi_{n}, t \mid \phi_{m}, t\right\rangle}{\left\langle\phi_{m}, t \mid \phi_{m}, t\right\rangle} \mathcal{A}_{m n}(t)\right) .
$$

Therefore, the complex and real geometric phase angles are related by

$$
\tilde{\gamma}_{m}(T)=\gamma_{m}(T)+\sum_{n \neq m} \int_{0}^{T} \frac{\left\langle\phi_{n}, t^{\prime} \mid \phi_{m}, t^{\prime}\right\rangle \mathcal{A}_{m n}\left(t^{\prime}\right) d t^{\prime}}{\left\langle\phi_{m}, t^{\prime} \mid \phi_{m}, t^{\prime}\right\rangle} .
$$

Next we observe that according to Eqs. (12), (25), and (27) the difference between $\tilde{\mathcal{A}}_{m}$ and $\mathcal{A}_{m}$ consists of terms which are bounded by $\eta$. Therefore, in the extreme adiabatic limit $\eta \rightarrow 0$, the two phase angles coincide. This in turn shows that in this limit the imaginary part of the complex geometric phase angle $\gamma_{m}(T)$ tend to zero. In practice, however, $\eta$ has a small but nonzero value (unless in the trivial case where the energy eigenvectors are stationary and $\left.\gamma_{m}(T)=0=\tilde{\gamma}_{m}(T)\right)$. In this case, $\gamma_{m}(T) \neq \tilde{\gamma}_{m}(T)$. This is consistent with the fact that $\gamma_{m}(T)$ is not necessarily real.

In the remainder of this paper we shall apply our general results to study the two-level system. This system has been the subject of detailed study [18, 19, 13] for its physical 
applications in particular in connection with the spontaneous decay of the excited states of atoms 19. .

Consider a two-dimensional Hilbert space where the Hamiltonian is a possibly nonHermitian $2 \times 2$ complex matrix with distinct eigenvalues. Setting $m=2$ in Eqs. (15) and using Eqs. (22), one has

$$
\begin{aligned}
& |\psi(t)\rangle=\tilde{C}(t)\left[\tilde{C}_{1}(t)\left|\psi_{1}, t\right\rangle+\left|\phi_{2}, t\right\rangle\right], \\
& \dot{\tilde{C}}_{1}+Q(t) \tilde{C}_{1}=\mathcal{R}(t),
\end{aligned}
$$

where

$$
\begin{aligned}
Q(t) & :=i\left[E_{1}(t)-E_{2}(t)\right]+\frac{\left\langle\psi_{1}, t \mid \dot{\psi}_{1}, t\right\rangle}{\left\langle\psi_{1}, t \mid \psi_{1}, t\right\rangle}-\frac{\left\langle\phi_{2}, t \mid \dot{\phi}_{2}, t\right\rangle}{\left\langle\phi_{2}, t \mid \phi_{2}, t\right\rangle}, \\
\mathcal{R}(t) & :=\frac{-i\left\langle\psi_{1}, t|H(t)| \phi_{2}, t\right\rangle}{\left\langle\psi_{1}, t \mid \psi_{1}, t\right\rangle} .
\end{aligned}
$$

In Eqs. (30) and the remainder of this paper, the adiabatic approximation is assumed to be valid and $\approx$ 's are replaced by $=$ 's.

Eq. (30) can be easily integrated to yield

$$
\tilde{C}_{1}(t)=W(t)\left(\tilde{C}_{1}(0)+\int_{0}^{t} \frac{\mathcal{R}\left(t^{\prime}\right)}{W\left(t^{\prime}\right)} d t^{\prime}\right)
$$

where

$$
W(t):=e^{-\int_{0}^{t} Q(s) d s} .
$$

Having obtained the general solution, one can easily check for the periodic solutions. In view of the fact that $Q$ and $\mathcal{R}$ are periodic functions of time with the same period $T$ as the Hamiltonian, one can show that

$$
\tilde{C}_{1}(t+T)-\tilde{C}_{1}(t)=W(t)\left(\tilde{C}_{1}(0)[W(T)-1]+W(T) \int_{0}^{T} \frac{\mathcal{R}\left(t^{\prime}\right)}{W\left(t^{\prime}\right)} d t^{\prime}\right) .
$$

Therefore, the initial condition leading to a periodic solution is given by

$$
\tilde{C}_{1}(0)=\frac{W(T) \int_{0}^{T} \frac{\mathcal{R}\left(t^{\prime}\right)}{W\left(t^{\prime}\right)} d t^{\prime}}{1-W(T)}
$$

If both the numerator and denominator on the right hand side of (34) vanish, then the right hand side of (33) vanishes and the solution (31) is always periodic. If the denominator 
vanishes, i.e.,

$$
W(T):=e^{i \int_{0}^{T}\left[E_{2}(s)-E_{1}(s)\right] d s} e^{-i \oint\left(\frac{i\left\langle\phi_{2}, R \mid d \phi_{2}, R\right\rangle}{\left\langle\phi_{2}, R \mid \phi_{2}, R\right\rangle}-\frac{i\left\langle\psi_{1}, R \mid d \psi_{1}, R\right\rangle}{\left\langle\psi_{1}, R \mid \psi_{1}, R\right\rangle}\right)}=1
$$

but the numerator does not, there is no periodic solutions. If the denominator does not vanish, $W(T) \neq 1$, then there is a particular initial condition given by Eq. (34) that leads to a periodic solution. It is for this solution that the formula (21) for the (cyclic) adiabatic geometric phase applies.

Clearly a similar treatment may be carried out for the choice $m=1$ in Eq. (15). The corresponding results are given by the same formulas as for the case $m=2$ except that one must interchange the labels 1 and 2 .

For the case of a Hermitian Hamiltonian where $\left|\phi_{n}, R\right\rangle=\left|\psi_{n}, R\right\rangle$, the right hand side of Eq. (22) vanishes and the trivial solution: $\tilde{C}_{n}=0$ is periodic. This solution corresponds to the well-known result that for an adiabatically changing Hamiltonian the eigenvectors $\left|\psi_{n}, 0\right\rangle$ of the initial Hamiltonian undergo cyclic evolutions. In this case, Eq. (21) gives Berry's phase [1].

Let us next consider the two-level system with the parametric Hamiltonian

$$
H=H[E, \theta, \varphi]:=E\left(\begin{array}{cc}
\cos \theta & e^{-i \varphi} \sin \theta \\
e^{i \varphi} \sin \theta & -\cos \theta
\end{array}\right),
$$

where $\theta \in \mathbb{R}$ and $E, \varphi \in \mathbb{C}$, [19]. Note that due to the form of the Hamiltonian (36), $\theta \in[0, \pi]$ and the real part $\varphi_{r}$ of $\varphi$ has the range $[0,2 \pi)$, whereas $E$ and the imaginary part $\varphi_{i}$ of $\varphi$ can take arbitrary complex and real values, respectively.

It is not difficult to show that the eigenvalues of $H$ are given by $\pm E$. Hence for $E \neq 0$ one has two distinct eigenvalues: $E_{1}=-E$ and $E_{2}=E$. The corresponding eigenvectors of $H$ and $H^{\dagger}$ are given by

$$
\begin{array}{ll}
\left|\psi_{1}\right\rangle=\left(\begin{array}{c}
-e^{-i \varphi} \sin \left(\frac{\theta}{2}\right) \\
\cos \left(\frac{\theta}{2}\right)
\end{array}\right), \quad\left|\psi_{2}\right\rangle=\left(\begin{array}{c}
\cos \left(\frac{\theta}{2}\right) \\
e^{i \varphi} \sin \left(\frac{\theta}{2}\right)
\end{array}\right), \quad \text { and } \\
\left|\phi_{1}\right\rangle=\left(\begin{array}{c}
-e^{-i \varphi^{*}} \sin \left(\frac{\theta}{2}\right) \\
\cos \left(\frac{\theta}{2}\right)
\end{array}\right), \quad\left|\phi_{2}\right\rangle=\left(\begin{array}{c}
\cos \left(\frac{\theta}{2}\right) \\
e^{i \varphi^{*}} \sin \left(\frac{\theta}{2}\right)
\end{array}\right),
\end{array}
$$

respectively. The geometric phase angles $\gamma_{m}(T)$ and $\tilde{\gamma}_{m}(T)$ can be easily computed,

$$
\gamma_{1}(T)=-\gamma_{2}(T)=\frac{1}{2} \oint(1-\cos \theta) d \varphi
$$




$$
\tilde{\gamma}_{1}(T)=\oint \frac{d \varphi_{r}}{1+e^{2 \varphi_{i}} \cot ^{2}\left(\frac{\theta}{2}\right)}, \quad \tilde{\gamma}_{2}(T)=-\oint \frac{d \varphi_{r}}{1+e^{-2 \varphi_{i}} \cot ^{2}\left(\frac{\theta}{2}\right)}
$$

Let us next consider the case where $E, \theta$ and $\varphi_{i}$ are constant and $\varphi_{r}=\omega t$. In this case, the Hamiltonian (36) is a non-Hermitian analogue of the Hamiltonian of a magnetic dipole interacting with a precessing magnetic field [1, 16, 20]. For this system, one can easily evaluate the integrals in (39) and (40), and obtain

$$
\begin{aligned}
& \gamma_{1}(T)=-\gamma_{2}(T)=\pi(1-\cos \theta) \\
& \tilde{\gamma}_{1}(T)=\frac{2 \pi}{1+e^{2 \varphi_{i}} \cot ^{2}\left(\frac{\theta}{2}\right)}, \quad \tilde{\gamma}_{2}(T)=\frac{-2 \pi}{1+e^{-2 \varphi_{i}} \cot ^{2}\left(\frac{\theta}{2}\right)}
\end{aligned}
$$

where $T:=2 \pi / \omega$.

Furthermore, one can obtain the explicit form of the initial condition for which the state vector (29) performs an adiabatic cyclic evolution. Using Eqs. (34), (37), and (38), one finds

$$
\tilde{C}_{1}(0)=-\frac{\sinh \varphi_{i} \sin \theta}{1+\left(\frac{\pi}{E T}\right)\left(\frac{e^{2 \varphi_{i}}-\cot ^{2}(\theta / 2)}{e^{2 \varphi_{i}+\cot ^{2}(\theta / 2)}}\right)} .
$$

One can repeat the above analysis for the state vector

$$
|\psi(t)\rangle=\tilde{C}(t)\left[\tilde{C}_{2}(t)\left|\psi_{2}, t\right\rangle+\left|\phi_{1}, t\right\rangle\right]
$$

which corresponds to the choice $m=1$ in Eqs. (14) - (23). In this case the initial condition leading to an adiabatic cyclic evolution is given by

$$
\tilde{C}_{2}(0)=-\frac{\sinh \varphi_{i} \sin \theta}{1+\left(\frac{\pi}{E T}\right)\left(\frac{e^{-2 \varphi_{i}}-\cot ^{2}(\theta / 2)}{e^{-2 \varphi_{i}}+\cot ^{2}(\theta / 2)}\right)} .
$$

It is not difficult to see that for $\varphi_{i}=0$ the above results tend to those of Berry [1]. It is also remarkable that unlike $\gamma_{m}(T)$ the new geometric phase angles $\tilde{\gamma}_{m}(T)$ are sensitive to the imaginary part $\varphi_{i}$ of $\varphi$.

In conclusion, we wish to emphasize that the issue of the existence of (exact) cyclic states of arbitrary period for a general Hermitian Hamiltonian is addressed in Ref. [21]. The results of [21] also generalize to the non-Hermitian Hamiltonians. This is simply because the existence of cyclic states of period $\tau$ is identical with the existence of eigenstates of the evolution operator $U(\tau)$. In the present article, we have considered the adiabatic cyclic states 
which have approximate cyclic evolutions. What we have established is the existence and construction of a class of adiabatic cyclic states which have the same period as the Hamiltonian but are not eigenstates of the initial Hamiltonian. These states have the same cyclicity properties as the well-known eigenstates of the initial Hamiltonian. The only difference is that the new adiabatic cyclic states acquire real geometric phase angles. For a two-level non-Hermitian Hamiltonian (36) we have constructed these states explicitly and computed the corresponding geometric phases. For this system if we choose $E, \theta$ and the imaginary part $\varphi_{i}$ of $\varphi$ to be constant and require the real part $\varphi_{r}$ of $\varphi$ to be proportional to time,

i.e., $\varphi_{r}(t)=\omega t$, then both the conventional geometric phase angle $\gamma_{m}(T)$ and the geometric phase angle $\tilde{\gamma}_{m}(T)$ introduced in this paper are real. However, unlike $\gamma_{m}(T), \tilde{\gamma}_{m}(T)$ depend on $\varphi_{i}$.

Finally we wish to note that one may follow the approach of Refs. [4, 5] to define a noncyclic analog of the adiabatic geometric phase obtained in the present paper. In this case, one is not limited to the periodic solutions of Eq. (22).

\section{References}

[1] M. V. Berry, Proc. Roy. Soc. London A 392, 45 (1984).

[2] Y. Aharonov and J. Anandan, Phys. Rev. Lett. 58, 1593 (1987)

J. Anandan and Y. Aharonov, Phys. Rev. D38, 1863 (1988).

[3] J. Samuel and R. Bhandari, Phys. Rev. Lett. 60, 2339 (1988);

J. Zak, Europhys. Lett., 9, 615 (1989);

A. K. Pati, Ann. Phys., 270, 178 (1998).

[4] G. G. de Polavieja and E. Sjöqvist, Am. J. Phys. 66, 431 (1998).

[5] A. Mostafazadeh, "Noncyclic Geometric Phase and Its Non-Abelian Generalization," J. Phys. A: Math. Gen., to appear.

[6] J. C. Garrison and E. M. Wright, Phys. Lett. A 128, 177 (1988). 
[7] F. Wilczek and A. Zee, Phys. Rev. Lett. 52, 2111 (1984).

[8] R. Newton, Phys. Rev. Lett. 72, 954 (1994).

[9] J. H. Hannay, J. Phys. A: Math. Gen. 18, 221 (1985);

M. V. Berry, J. Phys. A: Math. Gen. 18, 15-27 (1985);

J. Anandan, Phys. Lett. A 129, 201 (1988).

[10] J. C. Garrison and R. Y. Chiao, Phys. Rev. Lett. 60, 165 (1988);

J. Anandan and P. O. Mazur, Phys. Lett. A 173, 116 (1993);

A. Mostafazadeh, J. Phys. A: Math. Gen., 31, 7829 (1998).

[11] G. Dattoli, R. Mignani, and A. Torre, J. Phys. A: Math. Gen. 23, 5795 (1990).

[12] Ch. Miniature, C. Sire, J. Baudon, and J. Bellissard, Europhys. Lett. 13, 199 (1990).

[13] A. Mondragón and E. Hernández, J. Phys. A: Math. Gen. 29, 2567 (1996).

[14] G. Nenciu, and G. Rasche, J. Phys. A: Math. Gen. 25, 5741 (1992).

[15] A. Mostafazadeh, Phys. Rev. A 55, 1653 (1997).

[16] A. Bohm, Quantum Mechanics: Foundations and Applications, third edition (SpringerVerlag, Berlin, 1993).

[17] E. L. Ince, Ordinary Differential Equations, (Dover, New York, 1956).

[18] G. Dattoli, A. Torre, and R. Mignani, Phys. Rev. A 42, 1467 (1990).

[19] A. Kvitsinsky and S. Putterman, J. Math. Phys. 32, 1403 (1991).

[20] A. Mostafazadeh, J. Math. Phys. 38, 3489 (1997).

[21] J. Anandan, Phys. Lett. A 133, 171 (1988). 